QUARTERLY OF APPLIED MATHEMATICS

VOLUME LXX, NUMBER 3

SEPTEMBER 2012, PAGES 517-537

S $0033-569 X(2012) 01316-9$

Article electronically published on May 24, 2012

\title{
ON GLOBAL MINIMIZERS FOR A VARIATIONAL PROBLEM WITH LONG-RANGE INTERACTIONS
}

\author{
BY \\ RUSTUM CHOKSI \\ Department of Mathematics and Statistics, McGill University, Montreal, Canada \\ Dedicated to Constantine M. Dafermos on the occasion of his 70th birthday
}

\begin{abstract}
Energy-driven pattern formation induced by competing short and longrange interactions is common in many physical systems. In these proceedings we report on certain rigorous asymptotic results concerning global minimizers of a nonlocal perturbation to the well-known Ginzburg-Landau/Cahn-Hilliard free energy. We also discuss two hybrid numerical methods for accessing the ground states of these functionals.
\end{abstract}

1. Introduction: Energy-driven pattern formation. The analysis of pattern formation in complex systems (both physical and biological) has long received attention in the applied mathematics and physics communities (see for example [50, 18]). A central viewpoint, emerging from the seminal work of Turing, has been that pattern formation can be captured via bifurcations off a homogeneous (thermal equilibrium) state, wherein patterns are classified according to linear instabilities of the homogeneous state. Even outside this thermal equilibrium, it is often the case that there is some nonconvex energy associated with the phenomenon, and the PDE models used are indeed variational; that is, they represent a gradient flow (with respect to some metric) of the energy.

In recent years, mathematicians trained in the modern calculus of variations have taken a different approach to pattern formation based upon methods which directly address minimization of an energy. One attempts to capture and analyze observed patterns via minimization of an energy defined over all possible patterns. The applications here are significant, from ferromagnets, ferrofluids, and superconductors to elastic materials (e.g. martensitic materials), biomembranes and block copolymers (cf. 32 and the references therein).

Received January 15, 2012.

2010 Mathematics Subject Classification. Primary 49S05; Secondary 49M30, 35K30, 35K55, 74N15.

Key words and phrases. Global minimizers, long-range interactions, diblock copolymers.

E-mail address: rchoksi@math.mcgill.ca 

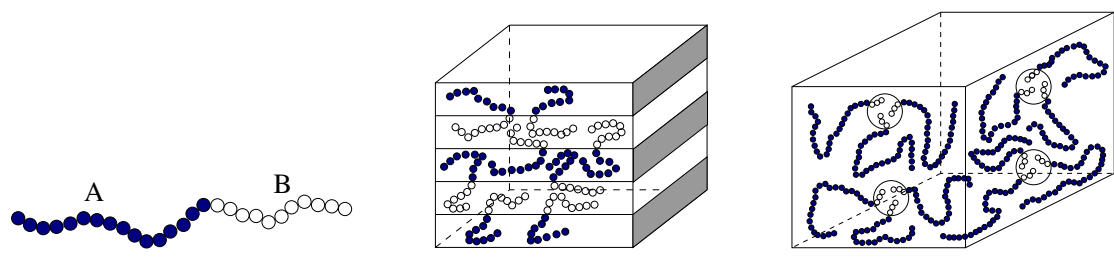

FIG. 1. A diblock copolymer macromolecule composed of two homogeneous chains of monomers $A$ and $B$ respectively, grafted together.

In this article we will address energy-driven pattern formation induced by competing short and long-range interactions (cf. 63]) where the interaction results in phase separation with an intrinsic length scale. We focus on a simple paradigm: a variational problem, originally used to model self-assembly of diblock copolymers, consisting of a nonlocal perturbation of a standard Cahn-Hilliard/Ginzburg-Landau energy. Clearly if one wants to address the energy landscape of a nonconvex functional with a goal of describing global minimizers, neither the local analysis around critical points, nor the solution of a gradient flow from any given state, is sufficient. Rather, we require new tools from nonlinear analysis and the calculus of variations supplemented by hybrid numerical methods which go beyond the integration of a PDE. This is certainly a formidable task. Consequently, we focus on our simple paradigm, and present a few rigorous results on global minimizers and a few numerical methods which attempt to access these ground states.

2. Self-assembly of diblock copolymers. Diblock copolymers are macromolecules composed of two chemically distinct homogeneous polymer chains (of monomer species A and B respectively) linked together by a covalent bond (see Figure 1). The thermodynamical incompatibility between the different subchains drives the system to phase separate; however, the covalent bonds between the different subchains prevent phase separation at a macroscopic length scale. As a result of these two competing trends, block copolymers undergo phase separation at a nanometer length scale, leading to an amazingly rich array of nanostructures. These structures present tremendous potentials for technological applications because they allow for the synthesis of materials with tailored mechanical, electrical, and chemical properties (see [5, 21, 29]). One of the main challenges is to describe and predict the possible nanostructures for a given set of molecular parameters such as the polymer architecture and monomer-monomer interactions. There are three dimensionless molecular parameters: (i) $\chi$, the Flory-Huggins interaction parameter which is inversely proportional to temperature and quantifies the incompatibility of the two monomer species; (ii) $N$, the index of polymerization which is simply the number of molecules in the polymer chain; (iii) $f$, the percentage of $A$ monomers in the total polymer chain.

The modeling of the self-assembly of these inhomogeneous polymers may seem to be straightforward as there are two interacting effects which need to be appropriately quantified and summed: 
- the chains like to be randomly coiled with Gaussian distribution (i.e. they behave like a Brownian motion sample path).

- part of the chain ( $A$ subchain) wants to separate from the other part ( $B$ subchain), without ever severing the covalent bond.

Ideally, a model should capture the self-assembled phase geometry depending on the material parameters. Based upon the statistical physics of polymer chains, analyzing such a model involves solving a highly nonlinear and nonlocal optimization problem - a very difficult problem for which approximations are needed. Most of the theoretical studies of block copolymers are based on a framework termed the self-consistent field theory (SCFT) 21. The SCFT of polymers is a field-theoretical representation of the statistical mechanics of polymers. It transforms the formidable task of integrating contributions to the partition function from many-chain interactions to the more manageable computation of the contribution of one polymer in a self-consistent field. This results in a free energy defined over monomer densities $\rho_{A}$ and $\rho_{B}$ and external (self-consistent) fields. The monomer densities and external fields are coupled via modified diffusion equations from the Feynman-Kac theory. Spectral-based numerical implementations of this theory have been successful (cf. [41, 17]) in producing a phase diagram in the $\chi N$ vs. $f$ plane which compares favorably with the experimental phase diagram of polyisoprene-polystyrene 31 .

A much simpler and direct phase field model was introduced by Ohta-Kawasaki in [48. Following Leibler [39], they invoked a Landau-type expansion in terms of monomer densities and derived a Landau-type free energy functional. As noted in [4], the OhtaKawasaki model gives rise to a nonlocal (of Coulombic type) perturbation of the ubiquitous Cahn-Hilliard functional [6], which has been the generator of an immense body of analytical work in applied mathematics and nonlinear partial differential equations (cf. 20]). As noted in [14, one can derive this nonlocal Cahn-Hilliard functional from the SCFT by linearization about the disordered state; the monomer densities are coupled with the self-consistent external fields via a modified diffusion equation and, about the disordered state, one linearizes the resulting dependence of external fields on the monomer densities. This is sometimes called the random phase approximation. However simple, we now show that this functional has a tremendously rich mathematical structure in higher space dimensions 1 Because of the ubiquity of Coulombic-type interactions, the functional can model many physical systems. It may also simply be viewed as a paradigm for pattern formation induced by short and long-range interactions.

3. Nonlocal Cahn-Hilliard and isoperimetric problems, heuristics, and some simulations. We minimize the functional defined over $\Omega \subset \mathbb{R}^{n}$ :

$$
(\mathrm{NLCH}) \quad \int_{\Omega}\left(\frac{\epsilon^{2}}{2}|\nabla u|^{2}+\frac{\left(1-u^{2}\right)^{2}}{4}\right) d x+\frac{\sigma}{2} \int_{\Omega}|\nabla v|^{2} d x
$$

\footnotetext{
${ }^{1}$ It is the higher-dimensional analogue of a functional studied by Müller 43 as a toy problem to capture multiple scales.
} 

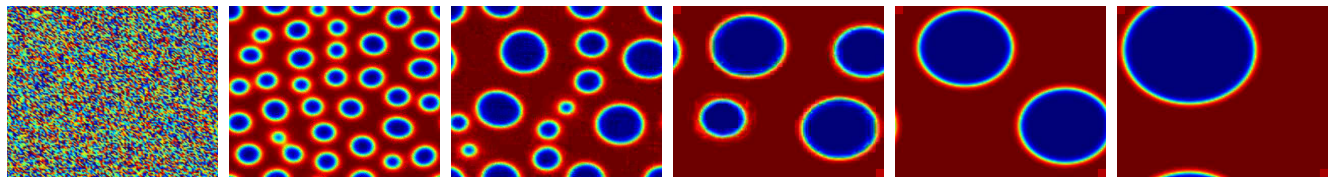

FIG. 2. Time series for the solution to the Cahn-Hilliard equation (i.e. (MCH) with $\sigma=0$ ) for a fixed value of $m$ and random initial conditions.
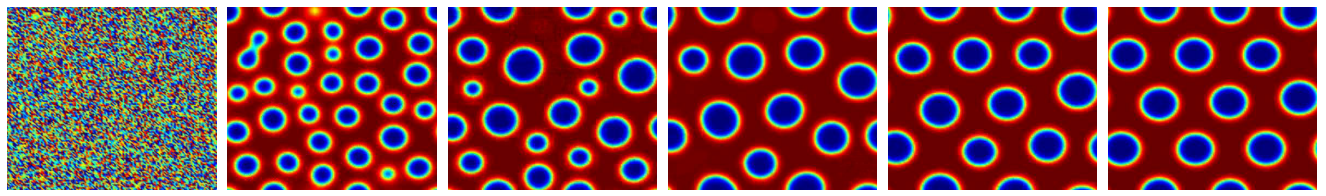

FIG. 3. Time series for the solution to (MCH) with $\sigma>0$ with the same value of $m$ and initial conditions. Computations performed by MSc student Ping Zhang at Simon Fraser University.

over the set

$$
\left\{u \in H^{1}(\Omega) \mid f_{\Omega} u=m,-\triangle v=u-m \text { in } \Omega \text { with either PBC or NBC }\right\} .
$$

Here, PBC and NBC refer to periodic and homogeneous Neumann boundary conditions, respectively. For the analysis of minimizers of $(\mathrm{NLCH})$, it is convenient to use both types of boundary conditions. For all simulations, we adopt periodic boundary conditions. In the context of diblock copolymers, the order parameter $u$ denotes the relative monomer density; i.e., regions where $u=1$ and $u=-1$ denote regions of pure $A$ and $B$ monomers respectively. Since we are modeling a system (a melt) of diblock copolymers of identical architecture, $m=2 f-1$. The parameters $\epsilon$ and $\sigma$ can also be related to $\chi, N, f$ (cf. 14 and Remark 3.11). If the monomer chains were not connected, their phase separation would be modeled by just the first two terms (the Cahn-Hilliard problem). The third nonlocal term is a, perhaps crude, way of capturing the connectivity of the chains.

To highlight the Coulombic-type long-range interactions, note that the nonlocal term may be written as

$$
\int_{\Omega}|\nabla v|^{2} d x=\int_{\Omega} \int_{\Omega} G(x, y)(u(x)-m)(u(y)-m) d x d y,
$$

where $G$ denotes the Green's function for $-\triangle$ with either periodic or Neumann boundary conditions. It is also convenient to view this as the $H^{-1}$ norm squared on the function $u-m$, i.e.

$$
\int_{\Omega}|\nabla v|^{2} d x=:\|u-m\|_{H^{-1}(\Omega)}^{2} .
$$

Whereas the minimization of the first two terms in $(\mathrm{NLCH})$ favors pure phases $(u=$ \pm 1 ) with minimal interfaces, the third nonlocal term favors the average $m$. Combining all three, minimization favors oscillations between the pure phases (with volume faction $m$ ) and a set number of interfaces dictated by $\epsilon$ and $\sigma$. The simplest heuristic (confirmed by simulations) is that minimizers will be periodic on an intrinsic length scale determined 

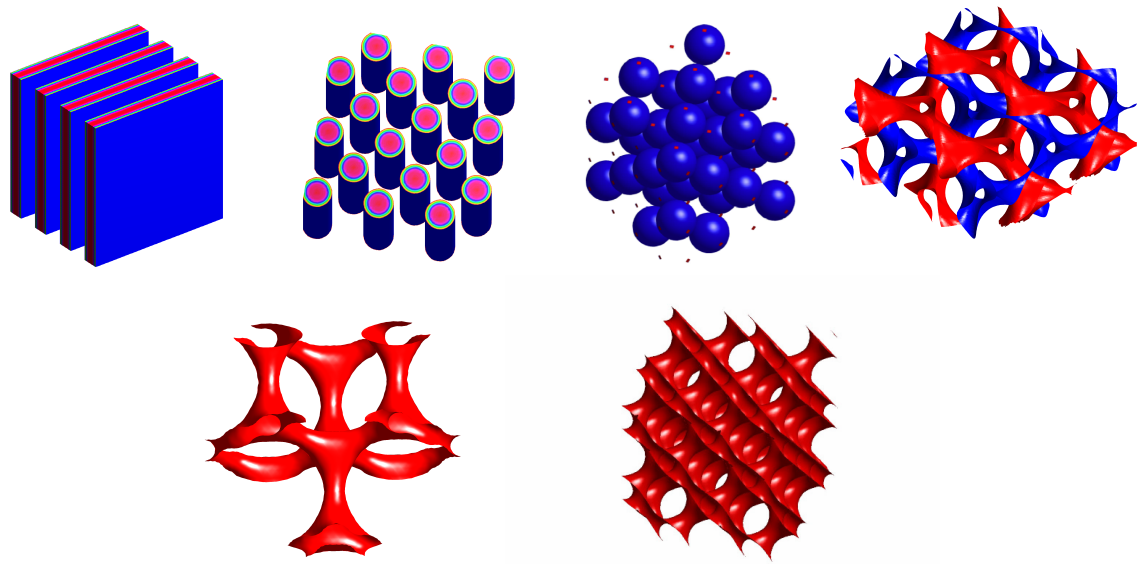

FIG. 4. Zero level sets of the final state for some sample 3D simulations attempting to access the ground state; $\mathrm{cf}$. 13 .

by $\epsilon$ and $\sigma$. Herein lies the fidelity of (NLCH) to the diblock copolymer problem, with the intrinsic scale being the consequence of the connectivity of the $A$ and $B$ subchains. Note that this connectivity is now imposed as a soft constraint via minimization rather than a hard constraint. The intrinsic length scale emulates the effective chain length of a single diblock macromolecule.

It is convenient to compute the gradient flow of (NLCH) with respect to the Hilbert space $H^{-1}$. In doing so we obtain the following modified Cahn-Hilliard equation:

$$
(\mathrm{MCH}) \quad u_{t}=\triangle\left(-\epsilon^{2} \triangle u-u+u^{3}\right)-\sigma(u-m) .
$$

Since we compute the gradient flow in the $H^{-1}$ norm, the presence of the nonlocal term in the functional (NLCH) simply gives rise to a local zeroth order perturbation of the well-known Cahn-Hilliard equation. However, as is illustrated in Figures 2 and 3 , this term favors $u=m$ and significantly changes the dynamics and steady states. Figure 2 shows the solution at different times for the Cahn-Hilliard equation (i.e. $\sigma=0$ ) with a fixed value of $m$, random initial conditions, and periodic boundary conditions. Figure 3 gives the analogous picture for $\sigma>0$ wherein an intrinsic length scale, independent of the domain size, between the drops is eventually set. Note that for all simulations we adopt periodic boundary conditions and deliberately take the domain size to be much larger than this intrinsic length.

The precise geometry of the interfacial region will depend on $m$, and the range of possibilities in 3D is significantly larger than in 2D. Numerical simulations suggest that minimizers are periodic on some fixed scale independent of domain size and, within a period cell, the structure appears to minimize surface area between the two phases. Thus in $3 \mathrm{D}$, the interface associated with minimizers resembles a triply periodic constant mean curvature surface. Sample 3D simulations attempting to access the ground state are shown in Figure 4. 
REMARK 3.1 (Rescalings). One can rescale either (NLCH) or $(\mathrm{MCH})$ to find that there are actually only two relevant parameters, $\epsilon \sqrt{\sigma}$ and $m$, needed to address the phase diagram. If one relates $\epsilon, \sigma, m$ to $\chi, N, f$ (cf. [14]), one finds that

$$
\frac{1}{\epsilon \sqrt{\sigma}} \sim \chi N
$$

REMARK 3.2 (Spectral analysis and local minimizers). An extensive spectral analysis of certain phase geometries has been carried out in a series of papers by Ren and Wei (see, for example, [54-[59]). This has yielded stability results not only for standard patterns like stripes, spots, rings, and spheres, but also, interestingly, for wiggled stripes and spots.

There is a parameter regime wherein the only global minimizer of (NLCH) is the uniform (disorder) state $u \equiv m$ (cf. [13]). Glasner [22] has given a detailed study of energy-minimizing equilibria and dynamics in a regime, close to the order-disorder transition, where the homogeneous state is stable.

If one focuses on the strong segregation limit, wherein the interfacial thickness of the phase boundaries tends to zero, the effective sharp interface problem (in the sense of $\Gamma$-convergence) can be written as (cf. [52, 14, 65]): For $\gamma>0$,

$$
\text { (NLIP) Minimize } \frac{1}{2} \int_{\Omega}|\nabla u| d x+\gamma \int_{\Omega}|\nabla v|^{2} d x
$$

over

$$
\left\{u \in B V(\Omega,\{-1,1\}) \mid \int_{\Omega} u=m,-\triangle v=u-m \text { in } \Omega \text { with either PBC or NBC }\right\} .
$$

In this formulation $u$ takes on only two values, and, hence, may be viewed as the characteristic function of a set of finite perimeter, the perimeter of which is the first term in the energy. The sole parameter $\gamma$ will now determine the length scale of the minimizers. Since this is a nonlocal perturbation of the well-known isoperimetric problem, we refer to this variational problem as the Nonlocal Isoperimetric Problem.

Note that by the Direct Method in the Calculus of Variations, there exists at least one minimizer of (NLCH) and (NLIP).

4. An intrinsic length scale for global minimizers based upon energy distribution. We address the periodicity of minimizers on an intrinsic length scale set by the coefficients in the functionals. Naturally, the domain size and boundary may not be exactly in sync with a given intrinsic period and, thus, even on a flat torus, an exact periodic structure would need to "slightly" adjust its period length. Moreover the shape of the domain boundary and the boundary conditions will have an effect, at least close to the boundary. Thus we will seek asymptotic results in a regime where the domain size is much larger than the intrinsic length. These results will not prove periodicity in any sharp form. Rather, for minimizers on cubes whose size is much larger than this intrinsic length scale, they will pertain to the uniform distribution of energy and density variations.

In $1 \mathrm{D}$, one may easily see why minimizers of (NLIP) should be periodic on an intrinsic length scale. Let $m=0$, set $\Omega=[0,1]$, and for any $u$ consider a primitive $w\left(u=w_{x}\right)$. 


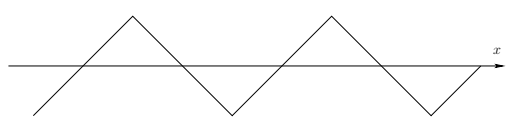

FIG. 5

The nonlocal term (the $H^{-1}$-norm squared of $u$ ) is now simply the $L^{2}$-norm squared of $w$. Thus the problem is to minimize:

$$
\int_{0}^{1}\left(\frac{1}{2}\left|w_{x x}\right|+\gamma w^{2}\right) d x
$$

over all saw-tooth functions with $w_{x}= \pm 1$. The second term by itself can be driven to zero with increasing oscillations in $w$ with equal volume faction. The first term penalizes such oscillations. The effect of the two is to set a fixed number of oscillations (see Fig. 51), and vanishing first variation of the energy will imply periodicity. Note also that the hard constraint

$$
\int_{0}^{1} w_{x} d x=0
$$

need not be imposed, as minimization of the energy will naturally choose such a function (i.e., it appears as a soft constraint). Similar results exist for the associated diffuse interface functional (cf. ([43, 53, 77, 70]).

In higher $\mathrm{D}$, we note:

- heuristically, the balancing of all the terms should also result in some form of periodicity - numerics support this;

- while there is no longer a primitive to work with, there is the analogous vector field $\mathbf{b}=\nabla v$.

Let us now focus on global minimizers (cf. Remark 4.1). Whether or not a global minimizer is exactly periodic remains open and, perhaps, debatable. We present a much weaker statement about periodicity based upon uniform energy distribution.

4.1. For the (NLIP). We consider (NLIP) with the homogeneous Neumann boundary condition and report on joint work with G. Alberti and F. Otto ([3]). The intrinsic length scale for minimizers is set entirely by $\gamma$. Since our result will only be meaningful for minimizers on domains of size larger than this intrinsic length, we take $\gamma=1$ and let $\Omega=Q_{L}$, the cube $[0, L]^{n}$. Consider a minimizer $u_{0}$ for (NLIP) on $Q_{L}(L \gg 1)$ and associated potential $v_{0}$ where

$$
-\triangle v_{0}=u_{0}-m \text { on } Q_{L}, \quad \nabla v_{0} \cdot \nu=0 \text { on } \partial Q_{L} .
$$

For $l>0$ and $a \in \mathbb{R}^{n}$, let $Q_{l}(a)$ denote the $n$-dimensional cube of side length $l$ centered at $a$. For any $Q_{l}(a) \subset Q_{L}$ define

$$
E\left(u_{0}, Q_{l}(a)\right):=\frac{1}{2} \int_{Q_{l}(a)}\left|\nabla u_{0}\right| d x+\gamma \int_{Q_{l}(a)}\left|\nabla v_{0}\right|^{2} d x,
$$

the restriction of the minimizer's energy to $Q_{l}(a)$. We have:

Theorem 4.1 ([3]). Fix the dimension $n$ and $m \in(-1,1)$. There exist positive constants $e_{0}, C_{1}, C_{2}$ such that: If $u_{0}$ is a minimizer of (NLIP) on $Q_{L}(L \gg 1)$, then for every $l$ such 
that $C_{1} \leq l \leq L$ and $Q_{l}(a) \subset Q_{L}$, we have

$$
\left|\frac{E\left(u_{0}, Q_{l}(a)\right)}{l^{n}}-e_{0}\right| \leq \frac{C_{2}}{l}
$$

Moreover, a similar statement holds separately for each term in the energy.

Note that the constants depend on both the space dimension $n$ and volume fraction $m$. These estimates tell us that the energy associated with a minimizer is uniformly distributed on an intrinsic scale up to an error. The error is optimal in the following sense: if the minimizer was exactly periodic and $e_{0}$ was the energy per unit volume on the entire domain, then a similar estimate would hold with the same $l^{-1}$ decay rate.

In proving Theorem 4.1, a key estimate is that the gradient of the optimal potential $\nabla v_{0}$ is bounded in $L^{\infty}$, independent of the domain size $L$. It is further proved in 3 . that $v_{0}$ is also uniformly bounded in $L^{\infty}$. These bounds can be used to give another statement supporting the notion of periodicity based upon the decay of large-scale density variations. To this end, consider any admissible $u$ for (NLIP) on $Q_{L}$ for $L \gg 1$. Let $a \in \mathbf{R}^{\mathbf{n}}, B_{R}(a) \subset Q_{L}$, and define $\phi_{R}(x):=\frac{1}{R^{n}} \phi\left(\frac{x-a}{R}\right)$ with $\phi$ a standard mollifier with support on $B_{1}(0)$. As our measure of large scale density variations, we consider the decay in $R$ of

$$
\int_{B_{R}(a)} \phi_{R}(x)(u(x)-m) d x
$$

One can readily check that if $u$ is exactly periodic, then $(*)$ decays exponentially fast. Thus one can quantify a notion of almost periodicity via the rate of decay. If $\left(u_{0}, \mathbf{b}_{0}\right)$ is the minimizer, then integrating by parts and using these $L^{\infty}$ bounds, we find

$$
\begin{aligned}
\left|\int_{B_{R}(a)} \phi_{R}(x)\left(u_{0}(x)-m\right) d x\right| & =\left|\int_{B_{R}(a)} \phi_{R}(x)\left(-\Delta v_{0}\right) d x\right| \\
& =\left|\int_{B_{R}(a)} \nabla \phi_{R}(x) \cdot \nabla v_{0} d x\right| \\
& =\left|\int_{B_{R}(a)}\left(-\Delta \phi_{R}(x)\right) v_{0} d x\right| \\
& \leq C \int_{B_{R}(a)}\left|\triangle \phi_{R}(x)\right| d x \\
& \leq C \frac{1}{R^{2}} \int_{B_{1}(a)}|\triangle \phi(x)| d x \\
& \leq C \frac{1}{R^{2}},
\end{aligned}
$$

where $C$ is a constant which depends only on $n$ and $m$. 
The proof of Theorem 4.1 is based upon the following reformulation of (NLIP). We note that if $v$ solves (4.2), then

$$
\begin{aligned}
& \int_{Q_{L}}|\nabla v|^{2} d x= \\
& \quad \min \left\{\int_{Q_{L}}|\mathbf{b}|^{2} d x \mid \mathbf{b} \in L^{2}\left(Q_{L}, \mathbb{R}^{n}\right), \operatorname{div} \mathbf{b}=u-m, \mathbf{b} \cdot \nu=0 \text { on } \partial Q_{L}\right\},
\end{aligned}
$$

where the minimum is attained only if $\mathbf{b}=\nabla v$. Hence, we consider the following reformulation of (NLIP):

$$
\min _{(u, \mathbf{b}) \in \mathcal{A}} F\left(u, \mathbf{b}, Q_{L}\right):=\int_{Q_{L}}|\nabla u|+\frac{1}{2} \int_{Q_{L}}|\mathbf{b}|^{2} d x
$$

where

$$
\begin{aligned}
& \mathcal{A}\left(Q_{L}\right):=\{(u, \mathbf{b}) \mid u \in B V\left(Q_{L}, \pm 1\right), \mathbf{b} \in L^{2}\left(Q_{L}, \mathbb{R}^{n}\right), \\
&\left.\operatorname{div} \mathbf{b}=u-m, \mathbf{b} \cdot \nu=0 \text { on } \partial Q_{L}\right\} .
\end{aligned}
$$

This reformulation has several advantages. For example, it localizes the functional and facilitates the use of cutting and pasting arguments. It further allows us to divorce from the particular choice of boundary conditions, here taken to be homogeneous Neumann. In fact, one could dispense entirely with the boundary conditions for $\mathbf{b} \cdot \nu$; as in the $1 \mathrm{D}$ case, the correct mean will be enforced as a soft, rather than a hard, constraint.

Theorem 4.1 has an identical statement for minimizers of $F\left(u, \mathbf{b}, Q_{L}\right)$ over $\mathcal{A}$. Its proof combines many estimates. We note here that one of the key estimates for the cutting and pasting arguments pertains to finding a good upper bound for the optimal energy on a cube in terms of Neumann boundary data for $\mathbf{b}$. That is, given $g \in L^{2}\left(\partial Q_{l}\right)$, we consider

$$
\Sigma_{g}\left(Q_{l}\right):=\min F\left(u, \mathbf{b}, Q_{l}\right),
$$

where the minimum is taken over all admissible pairs $(u, \mathbf{b})$ with $\operatorname{div} \mathbf{b}=u-m$ and $\mathbf{b} \cdot \nu=g$ on $\partial Q_{L}$. We now relate $\Sigma_{g}\left(Q_{l}\right)$ to $\|g\|_{L^{2}\left(\partial Q_{l}\right)}$. A central difficulty lies in the lack of convexity associated with the nonconvex constraint $\operatorname{div} \mathbf{b}=u-m \in\{-1-m, 1-m\}$. To this end, we relax this constraint by dispensing of the order parameter $u$, focusing on the bulk energy, and simply requiring div $\mathbf{b} \in[-1-m, 1-m]$. This new relaxed problem, involving only $\mathbf{b}$, is convex and has a dual formulation which can be solved via a trace estimate. We use the optimal $\mathbf{b}_{*}$ to the relaxed problem to construct an admissible pair as follows: We replace the $[-1,1]$-valued function $\operatorname{div} \mathbf{b}+m$ by a $\{-1,1\}$-valued function $u$ that is piecewise constant on rectangles of diameter order 1 , and is of the same local volume fraction. The surface energy and the additional bulk energy are of the order $l^{n}$.

4.2. For the $(\mathrm{NLCH})$. Based upon the methods and reformulations used for (NLIP) in [3], Spadaro 65] proved results in a similar spirit for the full diffuse interface functional (NLCH). To be consistent with his statements, we now adopt periodic boundary conditions. We recall $(\mathrm{NLCH})$

$$
\int_{\Omega}\left(\frac{\epsilon^{2}}{2}|\nabla u|^{2}+\frac{\left(1-u^{2}\right)^{2}}{4}\right) d x+\frac{\sigma}{2} \int_{\Omega}|\nabla v|^{2} d x .
$$


One can prove that the minimum energy scales like $\epsilon^{2 / 3} \sigma^{1 / 3}([9,45,65])$. Also the intrinsic length scale of the minimizer is readily seen to scale like $(\epsilon / \sigma)^{1 / 3}$. Thus we now keep the sample size fixed, i.e.,

$$
\Omega=\mathbb{T}^{n}, \quad \text { the } n \text {-dimensional torus of unit volume, }
$$

and seek an estimate for the regime where $\epsilon / \sigma \ll 1$, i.e. where the number of "period" cells is very large. This estimate will tell us that on any subcube of side $l$ times $(\epsilon / \sigma)^{1 / 3}$, the energy of a minimizer is approximately equal to $\epsilon^{2 / 3} \sigma^{1 / 3}$ times the volume of the subcube.

For a given $\epsilon$ and $\sigma$, consider any minimizer $u_{\epsilon, \sigma}$ of $(\mathrm{NLCH})$ on $\mathbb{T}^{n}$ with associated potential $v_{\epsilon, \sigma}$, where

$$
-\triangle v_{\epsilon, \sigma}=u_{\epsilon, \sigma}-m \quad \text { on } \mathbb{T}^{n} .
$$

For any $Q_{l}(a) \subset \mathbb{T}^{n}$ define

$$
\begin{aligned}
E_{\epsilon, \sigma}\left(u_{\epsilon, \sigma}, Q_{l}(a)\right):=\int_{Q_{l}(a)}\left(\frac{\epsilon^{2}}{2}\left|\nabla u_{\epsilon, \sigma}\right|^{2}\right. & \left.+\frac{\left(1-u_{\epsilon, \sigma}^{2}\right)^{2}}{4}\right) d x \\
& +\frac{\sigma}{2} \int_{Q_{l}(a)}\left|\nabla v_{\epsilon, \sigma}\right|^{2} d x
\end{aligned}
$$

We have

Theorem $4.2(65])$. Fix the dimension $n$ and $m \in(-1,1)$. There exist positive constants $C_{1}, C_{2}$ and a constant $e_{\epsilon, \sigma}$ depending on $\epsilon$ and $\sigma$ such that if $\epsilon / \sigma \leq C_{1}, C_{1}^{-1} \leq l \leq$ $(\sigma / \epsilon)^{1 / 3}$, and $a \in \mathbb{T}^{n}$, we have

$$
\left|\frac{E_{\epsilon, \sigma}\left(u_{\epsilon, \sigma}, Q_{(\epsilon / \sigma)^{1 / 3} l}(a)\right)}{\epsilon^{2 / 3} \sigma^{1 / 3}\left((\epsilon / \sigma)^{1 / 3} l\right)^{n}}-e_{\epsilon, \sigma}\right| \leq C_{2}\left(\frac{1}{l}+\epsilon^{2 / 3} \sigma^{1 / 3}\right) .
$$

Moreover, there exists a constant $e_{0}^{*}$ such that

$$
\lim _{\epsilon / \sigma \rightarrow 0} e_{\epsilon, \sigma}=e_{0}^{*} .
$$

Note here that the constant $e_{0}^{*}$ will depend on $n$ and $m$ and the structure of the double-well energy. Via the Modica-Mortola Gamma-convergence theorem (cf. 2, 42]), one can relate our $e_{0}$ for the sharp interface energy to $e_{0}^{*}$ (cf. 65]). Here one rescales space based upon the length scale $(\epsilon / \sigma)^{1 / 3}$ and rewrites the energy in terms of the small parameter $\epsilon^{2 / 3} \sigma^{1 / 3}$ (cf. Remark 3.1).

Similar to the previous idea of addressing the decay of large-scale density variations via (4.3), a corollary of Theorem 4.2 presented in [65] is that, under the same hypothesis as in Theorem 4.2, there exists a constant $C_{3}$ such that each minimizer $u_{\epsilon, \sigma}$ satisfies

$$
\left|f_{Q_{(\epsilon / \sigma)^{1 / 3} l}(a)} u_{\epsilon, \sigma} d x-m\right| \leq \frac{C_{3}}{l} .
$$

REMARK 4.1 (Intrinsic scale for local minimizers). The proofs of Theorems 4.1 and 4.2 very much rely on the fact that we have a global minimizer. Given the weak nature of their statements, it is possible that a similar estimate holds for any stable critical point. 
An interesting question concerns whether or not stable critical points of (NLIP) correspond to local minimizers in the sense that they are minimal with respect to small $L^{1}$ perturbations. Recently, Acerbi, Fusco and Morini [1] have proven such a result. This notion of local minimality can be lifted up to the diffuse interface problem (NLCH) via Г-convergence [33, 1].

REMARK 4.2 (Periodicity in related discrete models). Periodicity of the ground state in many discrete models (in condensed matter physics) is also often expected but very difficult to prove. Some examples are the work of Theil on two-dimensional LennardJones crystallization [69, and work on long-range dipolar and short-range ferromagnetic interactions by Giuliani, Lebowitz, and Lieb [23, 24]. The later work relies on a notion of Reflection Positivity.

5. The regime of small volume fraction and isolating the effects of the long-range interactions. In the previous section we addressed the intrinsic length scale of global minimizers. Simulations (e.g. 67, 68, 13]) show that level sets of (diffuse) phase boundaries strongly resemble a triply-periodic surface of constant mean curvature. However, on a finite torus, the combination of long-range and boundary effects will dictate that phase boundaries of global and local minimizers will NOT, in general, have constant mean curvature (see [44, [55, 15]). This perturbation from constant mean curvature seems to be very small - too small for numerical detection. How can we address this rigorously? A natural regime to focus on is the regime of a small volume fraction wherein the minimizing phases resemble small spherical inclusions of one phase in a large sea of the other phase. This is often called the droplet regime, and we shall henceforth refer to these droplets as particles. Focusing on this regime will allow us to decompose the nonlocal effects into self-effects on the shape of a single particle and interaction effects between different particles.

5.1. A $\Gamma$-convergence based asymptotic decomposition. Here we discuss joint work with M. Peletier ([11, 12]). We adopt the periodic boundary conditions and, working in dimension $n=3$, we set the domain equal to $\mathbb{T}^{3}$, the 3 -dimensional torus of unit volume. For convenience let us use a double-well potential in $(\mathrm{NLCH})$ with wells at 0 and 1 , and rewrite $(\mathrm{NLCH})$ as

$$
\mathcal{E}(u):=\epsilon^{2} \int_{\mathbb{T}^{3}}|\nabla u|^{2} d x+\int_{\mathbb{T}^{3}} u^{2}(1-u)^{2} d x+\sigma\left\|u-f_{\mathbb{T}^{3}} u\right\|_{H^{-1}\left(\mathbb{T}^{3}\right)}^{2} .
$$

Note that $f=f_{\mathbb{T}^{3}} u$. We give an asymptotic description in the sense of $\Gamma$-convergence (cf. 4]) for $\mathcal{E}$ in a limit wherein

- $\epsilon \rightarrow 0$ and $f \rightarrow 0$ (suitably slaved)

- $\sigma$ is chosen such that the number of small particles remains $O(1)$.

We will see

- the weak convergence (as measures) of a minimizing sequence to a collection of weighted delta measures

- an asymptotic description for the energy retaining information on the limiting shapes/patterns. At the leading order this will yield a functional associated with each delta mass, which isolates the effect of the long-range interactions on a 
single particle. At the next order we see the Coulomb-like interaction functional on the delta masses which is responsible for the self-assembly of the particles. To this end we introduce a small parameter $\eta$ where

$$
\int_{\mathbb{T}^{3}} u=f=\eta^{3} M
$$

and set

$$
v:=\frac{u}{\eta^{3}}, \quad \sigma=\frac{\epsilon}{\eta^{3}}, \quad \widetilde{W}(v):=v^{2}\left(1-\eta^{3} v\right)^{2} .
$$

Note that the wells of $\widetilde{W}$ are 0 and $1 / \eta^{3}$, and the limiting (as $\eta \rightarrow 0$ ) total mass is set by $M$.

Rewriting $\mathcal{E}(u)$ in terms of $v$, we find:

$$
\epsilon \eta^{2}\left\{\eta\left(\epsilon \eta^{3} \int_{\mathbb{T}^{3}}|\nabla v|^{2}+\frac{\eta^{3}}{\epsilon} \int_{\mathbb{T}^{3}} \widetilde{W}(v)\right)+\eta\left\|v-f_{\mathbb{T}^{3}} v\right\|_{H^{-1}\left(\mathbb{T}^{3}\right)}^{2}\right\}
$$

where the $H^{-1}$-norm is defined by (3.1). The terms in the outer parentheses remain $O(1)$ as $\epsilon, \eta \rightarrow 0$. Thus we focus on

$$
E_{\epsilon, \eta}:=\eta\left(\epsilon \eta^{3} \int_{\mathbb{T}^{3}}|\nabla v|^{2}+\frac{\eta^{3}}{\epsilon} \int_{\mathbb{T}^{3}} \widetilde{W}(v)\right)+\eta\left\|v-f_{\mathbb{T}^{3}} v\right\|_{H^{-1}\left(\mathbb{T}^{3}\right)}^{2} .
$$

As in [11, 12, it is convenient to first let $\epsilon$ tend to zero and then address the $\eta$-asymptotics of the sharp interface limit ( $\Gamma$-limit). By the Modica-Mortola Theorem 2, 42, for $\eta$ fixed, the $\Gamma$-limit as $E_{\epsilon, \eta}$ is readily seen to be

$$
F_{\eta}(v):=\eta \int_{\mathbb{T}^{3}}|\nabla v|+\eta\left\|v-f_{\mathbb{T}^{3}} v\right\|_{H^{-1}\left(\mathbb{T}^{3}\right)}^{2} \quad \text { defined for } v \in B V\left(\mathbb{T}^{3},\left\{0,1 / \eta^{3}\right\}\right) .
$$

Note that $\min F_{\eta} \sim O(1)$ as $\eta \rightarrow 0$.

Let $X$ denote the space of Radon measures with the topology of weak $*$ convergence. For $\mu_{\eta}, \mu \in X, \mu_{\eta} \rightarrow \mu$ denotes weak $*$ measure convergence, i.e.

$$
\int_{\mathbb{T}^{3}} f d \mu_{\eta} \rightarrow \int_{\mathbb{T}^{3}} f d \mu
$$

for all $f \in C\left(\mathbb{T}^{3}\right)$. We use the same notation for functions, i.e. when writing $v_{\eta} \rightarrow v_{0}$, we interpret $v_{\eta}$ and $v_{0}$ as measures whenever necessary. We trivially extend the functional $F_{\eta}$ to all of $X$ by assigning it to be $+\infty$ outside its domain of definition. We then have

THEOREM 5.1 ([1]). Let

$$
F_{0}\left(v_{0}\right):= \begin{cases}\sum_{i \in I} f_{0}\left(m_{i}\right) & \text { if } v_{0}=\sum_{i \in I} m_{i} \delta_{x_{i}} \\ \infty & \text { otherwise }\end{cases}
$$

where the localized energy for mass weight $m_{i}$ is given by 2

$$
f_{0}\left(m_{i}\right):=\inf \left\{\int_{\mathbf{R}^{3}}|\nabla w|+\int_{\mathbf{R}^{3}} \int_{\mathbf{R}^{3}} \frac{w(x) w(y)}{4 \pi|x-y|} d x d y \mid w \in\{0,1\}, \int_{\mathbf{R}^{3}} w=m_{i}\right\} .
$$

\footnotetext{
${ }^{2}$ The second long-range term may be viewed as the $H^{-1}$-norm squared on all of the space, i.e., $\|w\|_{H^{-1}\left(\mathbf{R}^{3}\right)}^{2}$
} 


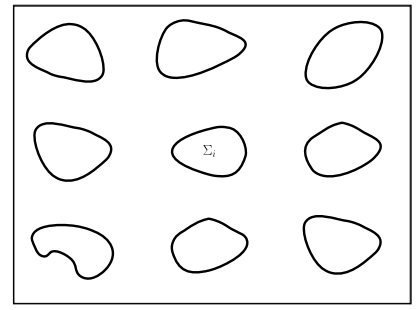

FIG. 6

Then, within the space $X$, we have $F_{\eta} \stackrel{\Gamma}{\longrightarrow} F_{0}$.

By $F_{\eta} \stackrel{\Gamma}{\longrightarrow} F_{0}$, we mean:

- (The lower bound and compactness) Let $v_{\eta}$ be a sequence in $X$ with fixed mass $\int_{\mathbb{T}^{3}} v_{\eta}$ and bounded energy $F_{\eta}\left(v_{\eta}\right)$. Then (up to a subsequence) $v_{\eta} \rightarrow v_{0}$, where the support of $v_{0}$ is countable and

$$
\liminf _{\eta \rightarrow 0} F_{\eta}\left(v_{\eta}\right) \geq F_{0}\left(v_{0}\right) .
$$

- (The upper bound) Let $v_{0} \in X$ with $F_{0}\left(v_{0}\right)<\infty$. Then there exists a sequence $v_{\eta} \rightarrow v_{0}$ such that

$$
\limsup _{\eta \rightarrow 0} F_{\eta}\left(v_{\eta}\right) \leq F_{0}\left(v_{0}\right) .
$$

The main technical difficulties associated with the proof lie in proving that, without loss of generality, any sequence with bounded energy and fixed (or simply bounded) mass must decompose into a collection of well-separated particles which converge to weighted delta measures (cf. Fig. 6). That is, we may assume that there is a finite collection of nonoverlapping sets $\Sigma_{i} \subset \mathbb{T}^{3}$ (of length scale $\sim \eta$ ) such that

$$
v_{\eta}=\sum v_{\eta}^{i}, \quad \text { where } \quad v_{\eta}^{i}=\frac{1}{\eta^{3}} \chi_{\Sigma_{i}} \quad v_{\eta} \rightarrow v_{0}=\sum m_{0}^{i} \delta_{x_{i}} .
$$

With this in hand, let us outline the basic step for the lower bound (see [11] for details). The energy now decomposes as follows:

$$
F_{\eta}\left(v_{\eta}\right)=\eta \sum_{i}\left\{\int_{\mathbb{T}^{3}}\left|\nabla v_{\eta}^{i}\right|+\int_{\mathbb{T}^{3}} v_{\eta}^{i} * G v_{\eta}^{i}\right\}+\eta \sum_{(i, j): i \neq j} \int_{\mathbb{T}^{3}} v_{\eta}^{i} * G v_{\eta}^{j},
$$

where $G$ is the Green's function of $-\triangle$ on $\mathbb{T}^{3}$. We now consider a mass-conservative rescaling that maps the amplitude to 1 ,

$$
w_{\eta}^{i}(x):=\eta^{3} v_{\eta}^{i}(\eta x)
$$

Rewriting (5.5) in terms of the $w_{\eta}^{i}$, we find the second sum to be $O(\eta)$. Writing the $G$ as the fundamental solution plus a corrector and letting $\eta \rightarrow 0$ yields $F_{0}$.

For the $\Gamma$-expansion, we can then subtract from the energy the leading-order term, renormalize (i.e. divide by $\eta$ ), and then let $\eta \rightarrow 0$. We then find (cf. [11]) a discrete Coulomb-like interaction term between the delta masses which is responsible for the self-assembly of the particles. 
In [12] we show how to modify the two parts of Theorem 5.1] to obtain the same $\Gamma$-limit for the diffuse interface functional in a diagonal limit in which both $\epsilon$ and $\eta$ tend to zero with a particular slaving of $\epsilon$ to $\eta$. To be brief, the lower bound involves a truncation argument. For the upper bound, we lift a sharp interface sequence to a diffuse interface sequence via a suitable quantification of Modica-Mortola construction in [42, 2].

REMARK 5.1 ( $\Gamma$-convergence and the energy landscape). De Giorgi's definition of $\Gamma$-convergence (including the associated compactness statement) immediately implies convergence of global minimizers. However, since its inception, it has proven to be a rather robust notion, retaining much of the general energy landscape structure from the point of view of local minimizers, critical points, and even certain gradient flow dynamics (see for example [33, 62, 30]).

REMARK 5.2 (Related asymtoptic descriptions). A different $\Gamma$-convergence study in the small volume fraction regime has recently been done by Goldman, Muratov and Serfaty [25, 26]. They work in 2D and focus on a regime associated with macroscopically large domains wherein the number of particles is not fixed. Within this regime they give an asymptotic, $\Gamma$-convergence based description of the energy. Working in 2D allows them to exploit some rather strong results of Muratov [45], which gives a description of global minimizers in terms of nearly round droplets.

Recently Cicalese and Spadaro [16] have given a rather detailed asymptotic description of the energy in (NLIP) in all space dimensions. They focus on regimes associated to a small volume fraction wherein the isoperimetric term is stronger than the nonlocal one and give a detailed description of the geometry of minimizers, focusing on a single droplet.

5.2. The single particle energy. We recall the leading order energy 3 of a single particle (droplet). For $a>0$, this is

$$
\inf \left\{\int_{\mathbb{R}^{3}}|\nabla w|+\int_{\mathbb{R}^{3}} \int_{\mathbb{R}^{3}} \frac{w(x) w(y)}{4 \pi|x-y|} d x d y \mid w \in\{0,1\}, \int_{\mathbb{R}^{3}} w=a\right\} .
$$

Note that the two terms in (5.6) are in direct competition: balls are best for the first term and worst for the second (the Coulombic potential). The latter point has an interesting history. Poincaré [51] considered the problem of determining possible shapes of a fluid body of mass $m$ in equilibrium. In a simplified form, this amounts to minimizing the total potential energy of a body (region) $E$ :

$$
\int_{E} \int_{E}-\frac{1}{C|x-y|} d x d y
$$

where $-(C|x-y|)^{-1}, C>0$ is the potential resulting from the gravitational attraction between two points $x$ and $y$ in the fluid. Poincaré showed that under some smoothness assumptions a body has the lowest energy if and only if it is a ball. It was not until almost a century later that the essential details were sorted via the rearrangement ideas of Steiner for the isoperimetric inequality. These ideas are captured in the Riesz Rearrangement Inequality and its development (cf. [38, 37]).

\footnotetext{
${ }^{3}$ This functional is also related to the Thomas-Fermi-Dirac-von Weizsäcker model for electrons (cf. 36, 40, ).
} 
So the two terms are in direct competition. We conjecture that the former wins, for $m_{0}$ below some threshold $m^{*}$, while the latter wins above this threshold. That is, we conjecture ([12]) that there exists $m^{*}>0$, such that for all $m_{0} \leq m^{*}$, there exists a unique global minimizer of (5.6), and it is a single ball of mass $m_{0}$. For $m_{0}>m^{*}$, a minimizer fails to exist. In fact, as $m$ increases past $m^{*}$, the ball remains a local minimizer, but a minimizing sequence consisting of two balls of equal size, that move away from each other, has lower limiting energy. This separation is driven by the $H^{-1}$ interaction energy, which attaches a positive penalty to any two objects at finite distance from each other. The limiting energy of such a sequence is thus simply the sum of the energies of two noninteracting balls. The results of [11] will imply the existence of a minimizer for $m_{0}$ sufficiently small; however with no explicit bound on $m_{0}$. Recently, Lu and Otto [40] have proved that for $m_{0}$ sufficiently large (again with no explicit bound), a minimizer fails to exist.

Proving that the only global minimizers of (5.6) are balls would provide rigorous support for why minimizers of $(\mathrm{NLCH})$ have phase boundaries which resemble periodic constant mean curvature surfaces; the nonlocal (long-range term) sets a periodic structure but within a period cell, the first two terms (associated with the perimeter) prevail.

We note that Knuepfer and Muratov 34 have recently addressed the analogue of (5.6) in 2D. There are also many interesting questions associated with local minimizers associated with (5.6) (see for example [46, 60]).

6. Some other results for global minimizers. Using a novel application of the modular function, Chen and Oshita 8 , prove that in $2 \mathrm{D}$, and under some assumptions on the admissible class, a hexagonal pattern is a ground state.

Sternberg and Topaloglu [66] have recently considered (NLIP) in the regime of small $\gamma$. They prove regularity of the phase boundary (for local minimizers) and prove that there is an interval of values for the mass constraint such that the global minimizer is exactly lamellar.

Peletier and Veneroni [49] study a related model with equal volume fraction $(m=0)$ wherein the nonlocal term is replaced with a Monge-Kantorovich distance. For the twodimensional system, they prove the following asymptotic result, for an appropriate small parameter $\epsilon$, supporting the statement that the ground state is periodic stripes. In the limit $\epsilon \rightarrow 0$, any sequence of patterns with uniformly bounded energy becomes stripelike; the pattern becomes locally one-dimensional and resembles a periodic stripe pattern of periodicity $O(\epsilon)$. In the limit, the stripes become uniform in width and increasingly straight.

They prove that their energy admits locally minimizing stripe patterns of width $O(\epsilon)$. As $\epsilon \rightarrow 0$, they prove that any admissible sequence with bounded energy must become stripe-like, and these stripes become increasingly straight and uniform in width.

Van Gennip and Peletier [27] also address global minimizers for related sharp interface functional modeling diblock-homopolymer blends. In 1D, they prove existence of minimizers of this functional and describe in detail the structure and energy of stationary points. Moreover, they characterize the conditions under which the minimizers may be 
nonunique. In higher dimensions they construct lower and upper bounds on the energy of minimizers, and explicitly compute the energy of spherically symmetric configurations.

7. Computationally accessing a global minimizer. For the simulation of minimizing structures, it is natural to focus on gradient descent, for example the $H^{-1}$ gradient flow (MCH) 4 Even though this gives rise to a stiff 4th-order PDE, many successful methods are readily available for its time integration (cf. for example [35, 19, 28]). In fact, several of these methods are unconditionally gradient stable with respect to timestepping.

On the other hand, simulations starting from random initial conditions, which eventually stabilize, are in no way guaranteed to stabilize in a global minimizer. The functional (NLCH) has many local minimizers and metastable states near which the dynamics are very slow. The latter is often called dynamic metastability and is characterized by evolution so slow that solutions appear to be stable. This metastable behavior can be misleading in the sense that after a long time, the solution undergoes drastic change. Numerically, one cannot distinguish a metastable state from a stable one, since they are both identified as solutions for which the relative change in $u-m$ or the energy between time-steps is smaller than some tolerance level. Techniques for dealing with metastability and highly nonconvex energy landscapes often belong to the broad class of statistical methods, called simulated annealing. They were created to navigate through a complex energy landscape in search of a global minimizer. A very simple form of simulated annealing can be achieved by adding unbiased noise to the evolved metastable state. This may force the solution out of the local minimizer that it is stuck in and make it continue its evolution through the energy landscape. Unfortunately, this approach does not provide a guaranteed way of addressing metastability as too much noise leads to the divergence of the solution and, even when the solution remains bounded, there is no way of ensuring that it will not revisit the local minimizers that it was stuck in before. Also, the added noise is very quickly damped out due to the 4th-order derivative term in this problem. We briefly present two examples of systematic methods which have proven fruitful.

7.1. Spectral weighting. We present results for a technique of spectral filtering implemented in joint work with Maras and Williams [10. Here our task was to simulate the ground state of $(\mathrm{NLCH})$ on $\mathbb{T}^{2}$ throughout parameter space. One of the methods employed was spectral weighting which is based upon the removal of insignificant spectral components from an evolved state. That is, we evolve the solution of $(\mathrm{MCH})$ from random initial conditions until a structure is formed, compute its Fourier coefficients, and keep only the modes which correspond to the coefficients above a certain threshold. The evolution is then continued and the process of spectral filtering repeated. Functional $(\mathrm{NLCH})$ can be thought of as a length selection mechanism and thus we evolve (NLCH) until past the point that the dominant length-scale has emerged. Denoting this length

\footnotetext{
${ }^{4}$ Alternatively one could consider the 2 nd-order $L^{2}$ gradient flow which can either be written as an integral-differential equation or a system of two differential equations. Here one would also have to numerically adjust for the required mass conservation.
} 

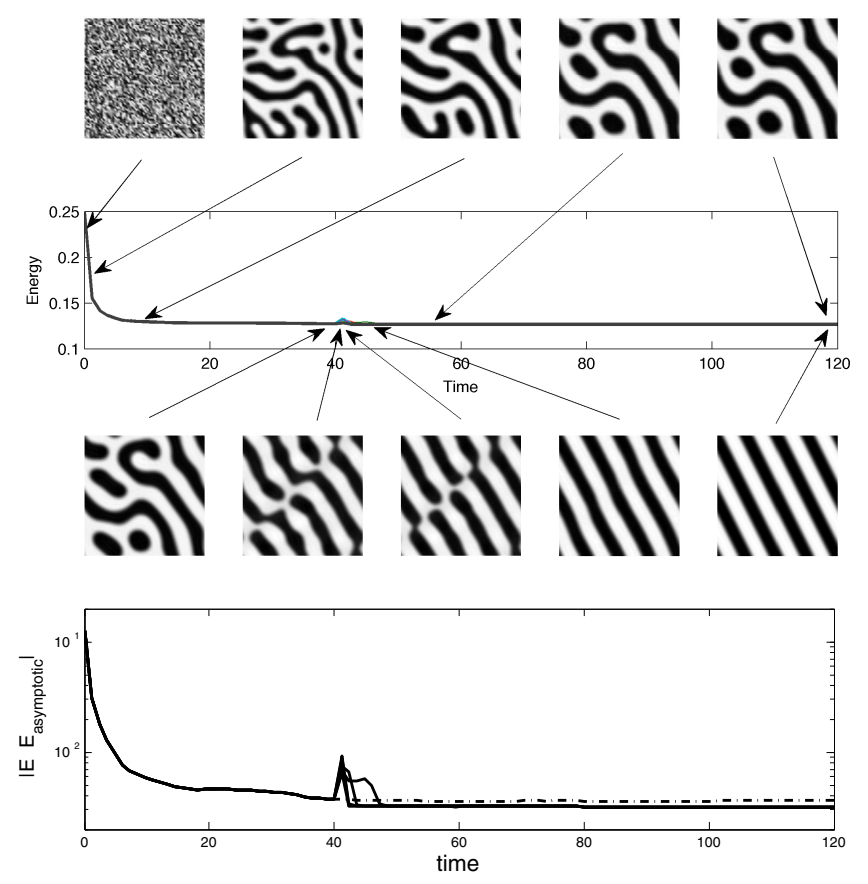

FIG. 7

scale by $k^{*}$, we damp the Fourier coefficients as follows: $\hat{u}(k) \rightarrow w\left(k ; k^{*}\right) \hat{u}(k)$, where

$$
\begin{aligned}
w\left(k ; k^{*}\right) & =(1-\rho)+ \\
\rho & \left(\exp \left(-5\left(1-|k| / k^{*}\right)^{2}\right)+\exp \left(-5\left(2-|k| / k^{*}\right)^{2}\right)+\exp \left(-5\left(3-|k| / k^{*}\right)^{2}\right)\right) .
\end{aligned}
$$

This keeps information at all wavelengths but focusses the dynamics at the key lengthscale and its higher harmonics. Choosing a good value for the parameter $\rho$ is rather ad hoc; experimentation indicates (i) there is little difference in the outcome with $0.05 \leq$ $\rho \leq 0.3$, (ii) with $\rho$ too small, there is no effect, and (iii) the "wrong" pattern may emerge if $\rho$ is too large or the standard gradient flow is not run long enough.

Figure 7 shows two runs for $(M C H)$ on $\mathbb{T}^{2}$ from the same initial conditions. The top has no spectral damping and ends up stuck in a metastable state whereas the bottom leads to straight stripes which we believe is the global minimizer. The energy of both runs is shown in the top of Figure 7 where the difference between the two runs is almost indistinguishable. A detailed view of the energy is presented at the bottom. See [10] for more details.

7.2. Motion by mean curvature. We present a simple illustration of motion by mean curvature as an annealing technique. This is work in progress with Shahriari and Ruuth 64] which numerically addresses $(\mathrm{NLCH})$ and $(\mathrm{MCH})$ on surfaces using the Closest Point Method of Ruuth and Merriman [61]. On any given surface, we combine the time integration of the $(\mathrm{MCH})$ with motion by mean curvature (viewing $u$ as a level set function) to arrive at low energy states. Motion by mean curvature focuses on minimizing the 

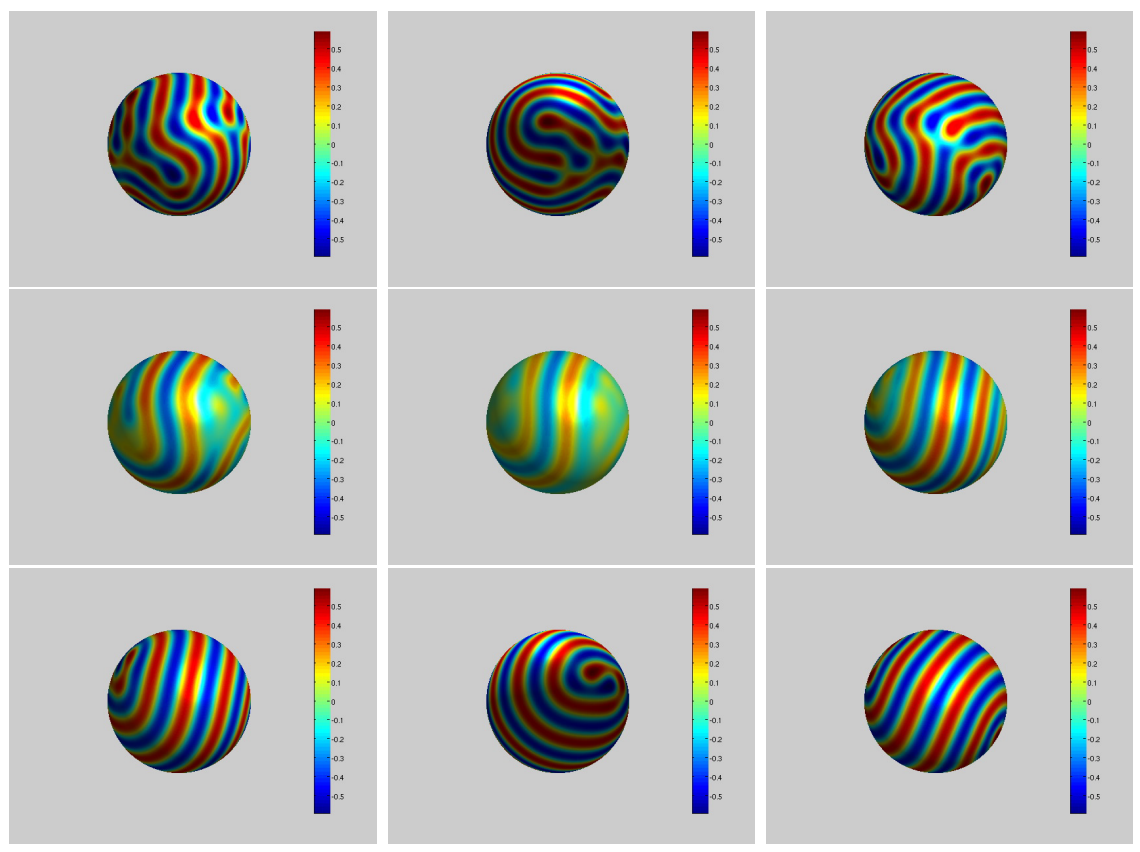

FIG. 8

first two terms in $(\mathrm{NLCH})$. Figure 8 illustrates this technique, working on the sphere in the lamellar regime. The top line shows a few late time shots (suitably rotated) of the evolution of $(\mathrm{MCH})$ from random initial conditions. These stabilize in a stripe pattern possessing several defects. We now apply motion by mean curvature with time shots illustrated in the second line; here the energy increases. Lastly, we again evolve according to $(\mathrm{MCH})$; however, this time the run stabilizes in a state of lower energy than had been previously reached. This final state is still a stripe pattern but with less curvature and, more importantly, fewer defects.

\section{REFERENCES}

[1] Acerbi. E., Fusco, N. and Morini, M.: Minimality via second variation for a nonlocal isoperimetric problem, preprint (2011).

[2] Alberti, G.: Variational Models for Phase Transitions, an Approach via $\Gamma$-convergence. Calculus of Variations and Partial Differential Equations (Pisa, 1996), 95-114, Springer, Berlin, 2000. MR 1757697

[3] Alberti, G., Choksi, R., and Otto, F.: Uniform Energy Distribution for Minimizers of an Isoperimetric Problem With Long-range Interactions. J. Amer. Math. Soc., 22-2, 569-605 (2009). MR2476783 (2009m:49073)

[4] Anzellotti, G. and Baldo, S: Asymptotic Development by Г-convergence. Appl. Math. Optim. 27, 105-123 (1993). MR1202527 (94b:49051)

[5] Bates, F.S. and Fredrickson, G.H.: Block Copolymers - Designer Soft Materials. Physics Today, 52-2, 32-38 (1999).

[6] Cahn, J.W. and Hilliard, J.E.: Free Energy of a Nonuniform System I. Interfacial Free Energy. J. Chem. Phys., 28(2), 258-267 (1958). 
[7] Chen, X. and Oshita, Y.: Periodicity and uniqueness of global minimizers of an energy functional containing a long-range interaction. SIAM J. Math. Anal. 37 no. 4, 1299-1332 (2005). MR2192296 (2006k:49027)

[8] Chen, X. and Oshita, Y.: An Application of the Modular Function in Nonlocal Variational Problems. Arch. Rat. Mech. Anal. 186-1, 109-132 (2007). MR2338353 (2009c:82060)

[9] Choksi, R: Scaling Laws in Microphase Separation of Diblock Copolymers. J. Nonlinear Sci. 11, 223-236 (2001). MR:1852942 (2003h:82091)

[10] Choksi, R., Maras, M., and Williams, J.F.: 2D Phase Diagram for Minimizers of a Cahn-Hilliard Functional with Long-range Interactions, SIAM J. Applied Dynamical Systems, 10-4, 1344-1362 (2011).

[11] Choksi, R. and Peletier, M.A.: Small Volume Fraction Limit of the Diblock Copolymer Problem: I. Sharp Interface Functional. SIAM J. Math. Anal., 42-3, 1334-1370 (2010). MR2653253 (2011j:49092)

[12] Choksi, R. and Peletier, M.A.: Small Volume Fraction Limit of the Diblock Copolymer Problem: II. Diffuse Interface Functional. SIAM J. Math Anal., 43-2, 739-763 (2011). MR 2784874

[13] Choksi, R., Peletier, M.A., and Williams, J.F.: On the Phase Diagram for Microphase Separation of Diblock Copolymers: an Approach via a Nonlocal Cahn-Hilliard Functional. SIAM J. Appl. Math. 69-6, 1712-1738 (2009). MR2496714 (2010f:74050)

[14] Choksi, R. and Ren, X.: On a Derivation of a Density Functional Theory for Microphase Separation of Diblock Copolymers. Journal of Statistical Physics, 113, 151-176 (2003). MR2012976 (2004k:82065)

[15] Choksi, R. and Sternberg, P.: On the First and Second Variations of a Nonlocal Isoperimetric Problem. J. Reine Angewandte Math. 611, 75-108 (2007). MR2360604 (2008j:49062)

[16] Cicalese, M. and Spadaro, E.N.: Droplet minimizers for a isoperimetric problem with long-range interactions, preprint.

[17] Cochran, E.W., Garcia-Cervera, C.J., and Fredrickson, G.H. Stability of the Gyroid Phase in Diblock Copolymers at Strong Segregation. Macromolecules 39(7), 2449-2451 (2006).

[18] Cross, M.C. and Hohenberg, P.C.: Pattern Formation Outside of Equilibrium. Reviews of Modern Physics 65:3, 851-1111 (1993).

[19] Eyre, D.: An Unconditionally Stable One-step Scheme for Gradient Systems. Unpublished paper, 1998 available at www.math.utah.edu/ eyre/research/methods/stable.ps

[20] Fife, P.: Models for Phase Separation and Their Mathematics. Electronic J. Diff. Equations, Vol. 2000 No. 48, 1-26 (2000). MR.1772733 (2001k:35147)

[21] Fredrickson, G.H.: The Equilibrium Theory of Inhomogeneous Polymers. Oxford Science Publications, Clarendon Press, 2006. MR2261726 (2008i:82153)

[22] Glasner, K: Spatially localized structures in diblock copolymer mixtures. SIAM J. Appl. Math. 70-6, 2045-2074 (2010). MR2630020 (2011e:82114)

[23] Giuliani, A., Lebowitz, J. L., and Lieb, E. H.: Striped phases in two dimensional dipole systems. Phys. Rev. B 76, 184426 (2007)

[24] Giuliani, A., Lebowitz, J. L., and Lieb, E. H.: Periodic minimizers in 1D local mean field theory. Comm. Math. Phys. 286 no. 1, 163-177 (2009). MR2470928(2009k:82036)

[25] Goldman, D., Muratov, C.B., and Serfaty, S: The Gamma-limit of the two-dimensional OhtaKawasaki energy. I. Droplet density, preprint (2011).

[26] Goldman, D., Muratov, C.B., and Serfaty, S: The Gamma-limit of the two-dimensional OhtaKawasaki energy. II. Droplet arrangement via the renormalized energy, preprint (2012).

[27] van Gennip, Y. and Peletier, M.A.: Copolymer-homopolymer Blends: Global Energy Minimisation and Global Energy Bounds, Calc. Var. 33, 75-111 (2008). MR2413102 (2009i:49008)

[28] Gomez, H. and Hughes, T.J.R.: Provably unconditionally stable, second-order time-accurate, mixed variational methods for phase-field models. J. Comp. Phys. 230, 5310-5327 (2011). MR2799512

[29] Hamley, I.W.: The Physics of Block Copolymers. Oxford Science Publications, 1998.

[30] Jerrard, R. L. and Sternberg, P.: Critical points via $\Gamma$-convergence: general theory and applications. J. Eur. Math. Soc. (JEMS) 11 no. 4, 705-753 (2009). MR2538502 (2010g:49017)

[31] Khandpur, A. K., Forster, S., Bates, F.S., Hamley, I.W. et al: Polyisoprene-Polystyrene Diblock Copolymer Phase Diagram near the Order-Disorder Transition. Macromolecules 28, 8796-8806 (1995). 
[32] Kohn, R.V.: Energy-driven Pattern Formation. Plenary Lecture and Proceedings of the International Congress of Mathematicians, Zürich, Vol. I, 359-383, Eur. Math. Soc., (2007). MR2334197 (2008k:49049)

[33] Kohn, R.V. and Sternberg, P.: Local Minimizers and Singular Perturbations. Proc. Roy. Soc. of Edinburgh Sect. A 111, 69-84 (1989). MR985990 (90c:49021)

[34] Knuepfer, H. and Muratov, C.B.: On an isoperimetric problem with a competing non-local term. I. The planar case. Submitted (2011).

[35] Kassam, A. and Trefethen, L. N.: Fourth-order Time-stepping for Stiff PDEs. SIAM J. Sci. Comput. 26, no. 4, 1214-1233 (2005). MR2143482 (2006g:65159)

[36] Lieb, E.H.: Thomas-Fermi and related theories of atoms and molecules, Rev. Mod. Phys. 53, 603641 (1981). MR629207 (83a:81080a)

[37] Lieb, E.H.: Existence and uniqueness of the minimizing solution of Choquard's nonlinear equation. Studies in Appl. Math., 57, 93-105 (1977). MR0471785 (57:11508)

[38] Lieb, E.H. and Loss, M.: Analysis. American Mathematical Society Graduate Studies in Mathematics Vol. 14. second edition (2001). MR1817225(2001i:00001)

[39] Leibler, L: Theory of Microphase Separation in Block Copolymers, Macromolecules 13, 1602-1617 (1980).

[40] Lu, J. and Otto, F.: Nonexistence of a Minimizer for the Thomas-Fermi-Dirac-von Weizsäcker model, preprint (2011).

[41] Matsen, M. W. and Schick, M.: Stable and Unstable Phases of a Diblock Copolymer Melt. Phys. Rev. Lett. 72, 2660-2663 (1994). Calc. Var. 1 169-204 (1993).

[42] Modica, L. and Mortola, S.: Un Esempio di $\Gamma$-Convergenza. Boll. Un. Mat. Ital. (5) 14-B 285-299 (1977). MR0445362(56:3704)

[43] Müller, S.: Singular perturbations as a selection criterion for periodic minimizing sequences. Calc. Var., 1, 169-204 (1993). MR.1261722 (95k:49030)

[44] Muratov, C.B.: Theory of Domain Patterns in Systems with Long-Range Interactions of Coulomb Type. Phys. Rev. E. 66, 066108 (2002). MR1953930 (2003k:82028)

[45] Muratov, C.B.: Droplet Phases in Non-local Ginzburg-Landau Models with Coulomb Repulsion in Two Dimensions, Commun. Math. Phys. 299, 45-87 (2010). MR2672798 (2011j:82092)

[46] Muratov, C.B. and Osipov, V.V.: General theory of instabilities for patterns with sharp interfaces in reaction-diffusion systems. Phys. Rev. E 53, 3101-3116 (1996). MR1388221 (96m:35164)

[47] Nishiura, Y. and Ohnishi, I.: Some Mathematical Aspects of the Micro-phase Separation in Diblock Copolymers. Physica D 84, 31-39 (1995). MR1334695 (96g:35196)

[48] Ohta, T. and Kawasaki, K.: Equilibrium Morphology of Block Copolymer Melts. Macromolecules 19, 2621-2632 (1986).

[49] Peletier, M.A. and Veneroni, M.: Stripe Patterns in a Model for Block Copolymers. Mathematical Models and Methods in Applied Sciences, 20, 843-907 (2010). MR2659741 (2011k:49024)

[50] Peletier, L.A. and Troy, W.C.: Spatial Patterns: Higher Order Models in Physics and Mechanics. Progress in Nonlinear Differential Equations and their Applications, 45. Birkhäuser, 2001. MR:1839555 (2002f:34106)

[51] Poincaré, H.: Sur une théorème de M. Liapunoff rélatif a l'équilibre d'une masse fluide. Comptes Rendus de L'Academie des Sciences, 104, 622-625 (1887).

[52] Ren, X., and Wei, J.: On the Multiplicity of Two Nonlocal Variational Problems, SIAM J. Math. Anal. 31-4, 909-924 (2000). MR1752422(2001a:74035)

[53] Ren, X., and Wei, J.: On Energy Minimizers of the Diblock Copolymer Problem, Interfaces and Free Boundaries 5, 193-238 (2003). MR1980472 (2004i:82077)

[54] Ren, X. and Wei, J.: Stability of Spot and Ring Solutions of the Diblock Copolymer Equation. J. Math. Phys. 45-11, 4106-4133 (2004). MR2098120 (2005h:82125)

[55] Ren, X. and Wei, J.: Wriggled Lamellar Solutions and their Stability in the Diblock Copolymer Problem. SIAM J. Math. Anal. 37-2, 455-489 (2005). MR2176111 (2006m:35112)

[56] Ren, X. and Wei, J.: Droplet solutions in the diblock copolymer problem with skewed monomer composition. Calc. Var. Partial Differential Equations. 25-3, 333-359 (2006). MR2201676(2007b:82107)

[57] Ren, X. and Wei, J.: Existence and Stability of Spherically Layered Solutions of the Diblock Copolymer Equation. SIAM J. Appl. Math. 66-3, 1080-1099 (2006). MR2216732 (2007a:34088)

[58] Ren, X. and Wei, J.: Single droplet pattern in the cylindrical phase of diblock copolymer morphology, Journal of Nonlinear Science, Vol. 17, No. 5, 471-503 (2007). MR2361417(2008i:82154) 
[59] Ren, X. and Wei, J.: Spherical Solutions to a Nonlocal Free Boundary Problem From Diblock Copolymer Morphology. SIAM J. Math. Anal. 39-5, 1497-1535 (2008). MR2377287(2009d:82168)

[60] Ren, X. and Wei, J.: A toroidal tube solution to a problem involving mean curvature and Newtonian potential, Interfaces and Free Boundaries, Vol. 13, No. 1, 127-154 (2011), MR2793855

[61] Ruuth, S.J. and Merriman, B.: A Simple Embedding Method for Solving Partial Differential Equations on Surfaces, J. Comput. Phys., 227(3): 1943-1961, 2008. MR2450979 (2009h:35239)

[62] Sandier, E. and Serfaty, S.: $\Gamma$-convergence of Gradient Flows with Applications to Ginzburg-Landau. Comm. Pure Appl. Math. 57-12, 1627-1672 (2004). MR2082242 (2005f:49002)

[63] Seul, M. and Andelman, D.: Domain Shapes and Patterns: The Phenomenology of Modulated Phases. Science 267476 (1995).

[64] Shahriari, B., Ruuth, S., and Choksi, R: Self-Assembly on General Surfaces via the Closest Point Method, in preparation.

[65] Spadaro, E.N.: Uniform Energy and Density Distribution: Diblock Copolymer Functional. Interfaces Free Bound. 11, 447-474 (2009). MR2546607(2011b:82097)

[66] Sternberg, P. and Topaloglu, I.: On the Global Minimizers of a Nonlocal Isoperimetric Problem in Two Dimensions. Interfaces and Free Boundaries, 13, no. 1, 155-169 (2011). MR2793856

[67] Teramoto, T. and Nishiura, Y.: Double Gyroid Morphology in a Gradient System with Nonlocal Effects. Journal of the Physical Society of Japan. Vol. 71-7, 1611-1614 (2002).

[68] Teramoto, T. and Nishiura, Y.: Morphological Characterization of the Diblock Copolymer Problem with Topological Computation. Japan Journal of Industrial and Applied Mathematics 27, 175-190 (2010). MR2720552

[69] Theil, F.: A Proof of Crystallization in Two Dimensions. Comm. Math. Phys. 262-1, 209-236 (2006). MR 2200888(2007f:82018)

[70] Yip, N.K.: Structure of Stable Solutions of a One-dimensional Variational Problem. ESAIM: Control, Optimisation and Calculus of Variations, 12-4, 721-751 (2006). MR2266815 (2007g:49027) 